\title{
Research on Anisotropic Characteristics of Rock and Intelligent Recognition of Precursory Signal
}

\author{
Nan Li $\mathbb{D}^{1},{ }^{1}$ Zhibo Zhang $\mathbb{D}^{2,3}$ Majid Khan, ${ }^{2,3}$ Shaohua Zhang, ${ }^{1}$ Xianan Liu, ${ }^{2,3}$ and Shujie Li ${ }^{2,3}$ \\ ${ }^{1}$ State Key Laboratory of Coal Resources and Safe Mining, School of Mines, China University of Mining and Technology, Xuzhou, \\ Jiangsu 221116, China \\ ${ }^{2}$ State Key Laboratory of High-Efficient Mining and Safety of Metal Mines, Ministry of Education, \\ University of Science and Technology Beijing, Beijing 100083, China \\ ${ }^{3}$ School of Civil and Resource Engineering, University of Science and Technology Beijing, Beijing 100083, China
}

Correspondence should be addressed to Zhibo Zhang; zhangzhibo@ustb.edu.cn

Received 20 December 2019; Revised 14 September 2020; Accepted 22 September 2020; Published 8 October 2020

Academic Editor: Guoyang Fu

Copyright $\odot 2020 \mathrm{Nan}$ Li et al. This is an open access article distributed under the Creative Commons Attribution License, which permits unrestricted use, distribution, and reproduction in any medium, provided the original work is properly cited.

In order to study the change in anisotropic characteristics of rock materials during the uniaxial compression process, an advanced ultrasonic experimental device and loading device are used to establish an experimental system for measuring ultrasonic waveform in three orthogonal directions in real-time. The experimental results show that there exists pronounced difference between ultrasonic receiving waveform in axial and radial directions. Based on the multifractal theory, multifractal spectrums of receiving waveform are calculated, and multifractal parameters $(\Delta \alpha$ and $\Delta f)$ are further analyzed. The multifractal spectrum in the radial direction gradually becomes smaller, but the multifractal spectrum in the axial direction presents an "increase-decrease" trend. During the entire uniaxial compression process, the anisotropic characteristics of the rock sample show an "enlarge-reduce" trend, which is caused by the development and growth of microcracks. Furthermore, a quantitative relationship between multifractal parameter $\Delta \alpha$ and stress $\sigma$ is proposed. It indicates that the parameters $\Delta \alpha$ and $\sigma$ satisfy the power function. On this basis, a method is proposed that ultrasonic waveform precursor characteristics are recognized automatically using the backpropagation (BP) neural network to monitor the damage state of the rock sample. The application results show that this method can provide an early warning signal more than 100 seconds before the rock sample buckling failure. This research can provide a useful reference for studying the anisotropic characteristics of the rock sample during the uniaxial compression process. The proposed method in this paper has essential meaning for monitoring and early warning of rock stability.

\section{Introduction}

Rock is a naturally occurring aggregate of various minerals in a solid mass possessing heterogeneous internal structure, and its physical and mechanical properties have important impacts on construction and operation of underground engineering [1-5]. In general, directly describing the change in the physical structure of a rock body is quite challenging and complex. Therefore, a number of indirect monitoring methods have been in development and practically in use for monitoring the stability state of the rock [6-9].

Ultrasonic is an acoustic wave with high directivity, which implies that it is capable of generating sound waves with better efficiency. The parameters of the ultrasonic acoustic wave have high sensitivity to the physical state of the medium that it passes through. Consequently, ultrasonic is one of the best and the most reliable monitoring methods for characterizing the degree of damage of the rock mass. Initially, ultrasonic velocity parameters attracted the attention of scholars. Birch [10], Nur [11], and Zhang et al. [12] studied the relationship between ultrasonic velocity and confining pressure, loading direction, and main fracture surface, respectively. With the continuous progress of experimental instruments and equipment, change in the ultrasonic waveform received much attention by the scientific community. Guo and $\mathrm{Fu}$ studied the change characteristics of coda attenuation under different effective stresses using laboratory measurement devices [13]. Wulff et al. studied 
wave attenuation characteristics during the microfracturing process and estimated the fracture group density using attenuation coefficient [14]. Cao et al. analyzed the relationship between porosity and elastic wave. They inferred from their findings that with the increase in porosity of the rock mass, the elastic wave decreased [15]. Toksoz et al. measured the attenuation characteristics of $P$-wave and $S$-wave in dry and saturated rocks. The results showed that attenuation coefficients increase linearly with frequency for both $P$-wave and $S$-wave in dry and saturated rock units [16].

Rock is an anisotropic material composed of various minerals possessing diverse macroscopic mechanical performance in different directions under various external stresses due to the complexity and randomness of the microstructure, the fabric, and heterogeneity in the rock. Therefore, when ultrasonic wave propagates through the rock subjected to the loading, there exists a pronounced difference in the ultrasonic waveform in different directions. Some research studies indicated that rock materials have fractal characteristics during the entire damage process [17-19] with a distinct fractal phenomenon for the physical signal produced by the deformation of the rock [20-25]. In order to take the advantage of this phenomenon and enlighten further comprehensions in this paper, an attempt has been made to apply the fractal theory for deeply studying the change characteristics of the ultrasonic waveform in three orthogonal directions which would directly help in describing the anisotropic characteristics of the rock during the uniaxial compression process. Based on the analysis of the obtained results, a new monitoring and early warning method is proposed to describe the damage state of the rock using the backpropagation (BP) neural network. This research has considerable benefits for understanding the mechanism of ultrasonic waveform anisotropy and possessing an important theoretical and practical significance for describing the anisotropic characteristics of the rock using ultrasonic waveform. It can provide a theoretical basis for better monitoring the stability state of the rock and early warning of the rock buckling failure using ultrasonic waveform.

\section{Experimental Preparation}

2.1. Experimental System. The experimental system consisted of a loading system, an ultrasonic transmitting, receiving system, and a shielding system. The loading system mainly included a YAW4306 electric-hydraulic serving press machine having a maximum load of $3 \mathrm{MN}$. The test force resolution and relative error were $1 / 300,000$ and $\pm 1 \%$, respectively. The press machine had two control modes, force control mode and displacement control mode, which can be used to perform various mechanical experiments. The ultrasonic signal transmitting terminal was an ARB-1410 arbitrary waveform generator produced by Physical Acoustics Corporation (PAC). This card can be used as an acoustic source generator for active ultrasonic or guided wave technology to obtain waveform, energy, and frequency. The ultrasonic signal receiving terminal was a 24-channel micro-II acoustic emission acquisition system, which included a system host, system software, and preamplifier. Both the transmitting and receiving transducers were $\alpha-15$ transducers with a peak frequency of $150 \mathrm{kHz}$. In order to reduce the influence of the interface between the transducers and the sample on the ultrasonic wave propagation, the coupling agent is used on the contact surface between the transducers and the sample to enhance coupling. The shielding effect of the shielding room was more than $85 \mathrm{~dB}$, which can effectively reduce electroacoustic interference.

2.2. Experimental Material. The rock samples used in the experiment were cut and polished to cube size with dimension $(150 \mathrm{~mm} \times 150 \mathrm{~mm} \times 150 \mathrm{~mm})$. Rock samples were collected from the large pieces of limestone. A total of fifteen rock samples were processed to conduct the designed experiment. Among them, the testing group includes twelve rock samples (from TR-1 to TR-12), and the validation group consists of three rock samples, namely, VR-1, VR-2, and VR-3.

2.3. Experimental Program. In this experiment, the loading mode of rock samples is uniaxial compression. According to the mechanical properties of the rock samples, the force control mode was used while the adopted loading rate was set as $1000 \mathrm{~N} / \mathrm{s}$.

Ultrasonic real-time monitoring was used in the experiment. As shown in Figure 1, three groups of transmitting and receiving transducers were arranged on three orthogonal directions. For transducers that are arranged parallel to the loading direction (indicated by green circles), protection mold is designed and used, to ensure that an external load did not damage the transducers. The time interval of the transmitted ultrasonic waveform was one second. Two seconds after the third transmitting transducer transmit waveform, the first transmitting transducer continued to transmit waveform. The transmitted wave is a sine wave with a main frequency of $110 \mathrm{kHz}$, as shown in Figure 2 . According to the Nyquist-Shannon sampling theorem, the sampling frequency was set as $10 \mathrm{MHz}$ to preserve complete information of the original signal, and the sampling length was $10 \mathrm{k}$ (10,240 points).

\section{Experimental Results Analysis}

Since the obtained results are similar, in this paper, only experimental results of TR-1 are presented for interpretation purpose. During the entire loading process, ultrasonic parameters in three orthogonal directions are recorded in realtime, as shown in Figure 3.

According to Figure 3, some differences among receiving waveforms in three orthogonal directions are evident. Overall, receiving waveforms in three orthogonal directions were similar prior to loading. However, there was a difference during the loading process.

In the $X$ direction, the amplitude of the waveform was large before stress reached $40 \%$ of the peak stress. Especially, when stress is in the range of $20 \%-30 \%$ of the peak stress, the main peak of receiving waveform was prominent, and 


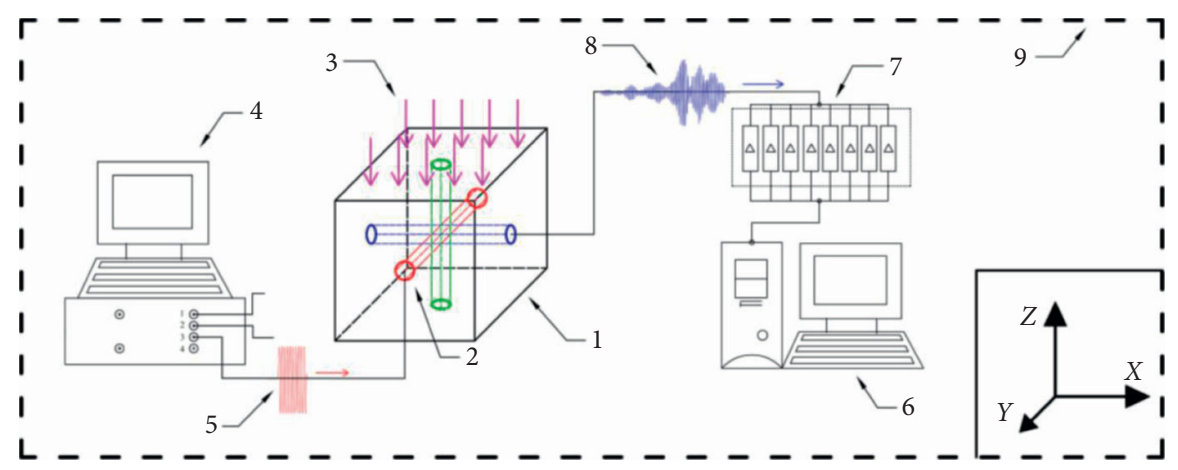

FIGURE 1: Schematic diagram of transducers arrangement. (1) The rock sample; (2) ultrasonic transducers; (3) press; (4) ultrasonic signal transmitting terminal; (5) transmitted sine signal; (6) ultrasonic signal receiving terminal; (7) preamplifier; (8) received signal; (9) shielding room.

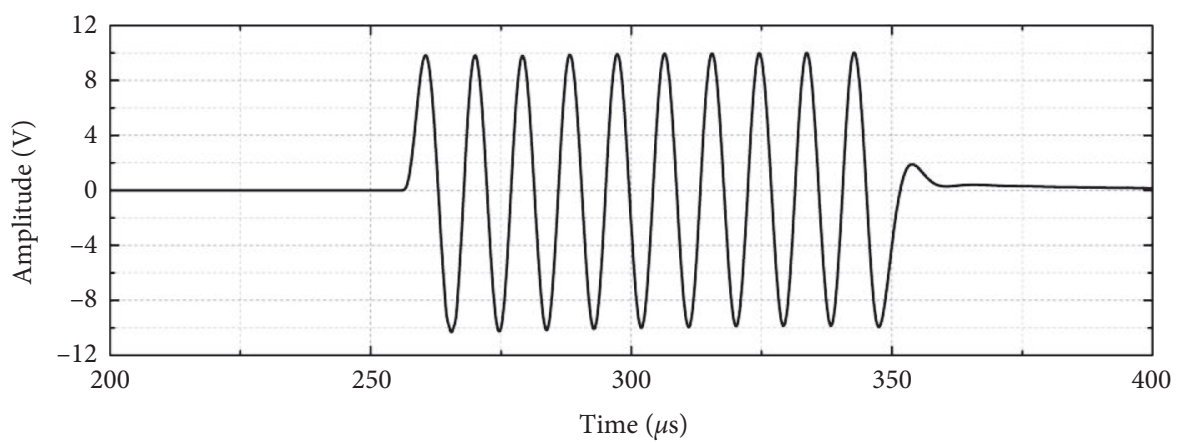

Figure 2: Transmitted waveform.

waveform presented a spindle shape. After stress exceeded $40 \%$ of the peak stress, the amplitude of the waveform gradually decreased as stress increases, and the main peak became less prominent. When stress reached $60 \%$ of peak stress, the amplitude of the waveform decreased obviously and reached the minimum value before the occurrence of the rock sample buckling failure. In the $Y$ direction, the change trend of receiving waveform was the same as in the $X$ direction, but the amplitude of the waveform is comparatively smaller than that in the $X$ direction.

Compared with receiving waveforms in the $X$ direction and the $Y$ direction, the change of receiving waveform in the $Z$ direction was very different. During the entire loading process, the amplitude of receiving waveform is large, and almost no considerable change is observed.

\section{Multifractal Analysis of Receiving Waveform}

4.1. Multifractal Theory. Based on the multifractal theory, the box-counting dimension method is used to calculate the multifractal characteristic of receiving waveform in three orthogonal directions in this part [26-29].

Assuming that the frequency spectrum series $\left[x_{i}\right]$ can be divided into many subsets whose length is $\varepsilon(\varepsilon<1)$, and the probability distribution of each subset is calculated as $P_{i}(\varepsilon)$. If the series meet multifractal characteristics, the probability distribution function $P_{i}(\varepsilon)$ meets the following equation:

$$
P_{i}(\varepsilon) \sim \varepsilon^{\alpha},
$$

where $\alpha$ is a constant known as the singularity exponent. It controls the singularity of probability function $P_{i}(\varepsilon)$, reflecting nonuniformity of a subset probability.

If the number of units with the same probability in the subsets are marked, then $\alpha$ is denoted as $N_{\alpha}(\varepsilon)$; generally, the smaller the divided scale $\varepsilon$ is, the more the number of subsets obtained. Hence, they have the following relationship:

$$
N_{\alpha}(\varepsilon) \propto \varepsilon^{-f(\alpha)},
$$

where $f(\alpha)$ represents the increase rate of the number of units in the $\alpha$ subset with $\varepsilon$ decreasing, that is, the fractal dimension of the $\alpha$ subset.

In the real calculation, utilization of the definition makes the calculation difficult. As an alternative, at present, the statistical physics method is applied to compute these multifractal parameters.

First, a partition function is defined as

$$
X_{q}(\varepsilon) \equiv \sum P_{i}(\varepsilon)^{q} \sim \varepsilon^{\tau(q)},
$$

where $\tau(q)$ is the quality index, with $-\infty<q<+\infty$. In real calculation, when $|q|$ reaches some definite value, the multifractal spectrum tends to be stable, followed by even greater values that possess no impact on multifractal spectrum. Generally, the value $q$ is defined within a specific range.

As equation (3) holds true, that is, defined partition function $X_{q}$ is the power function of the divided scale $\varepsilon, \tau(q)$ 

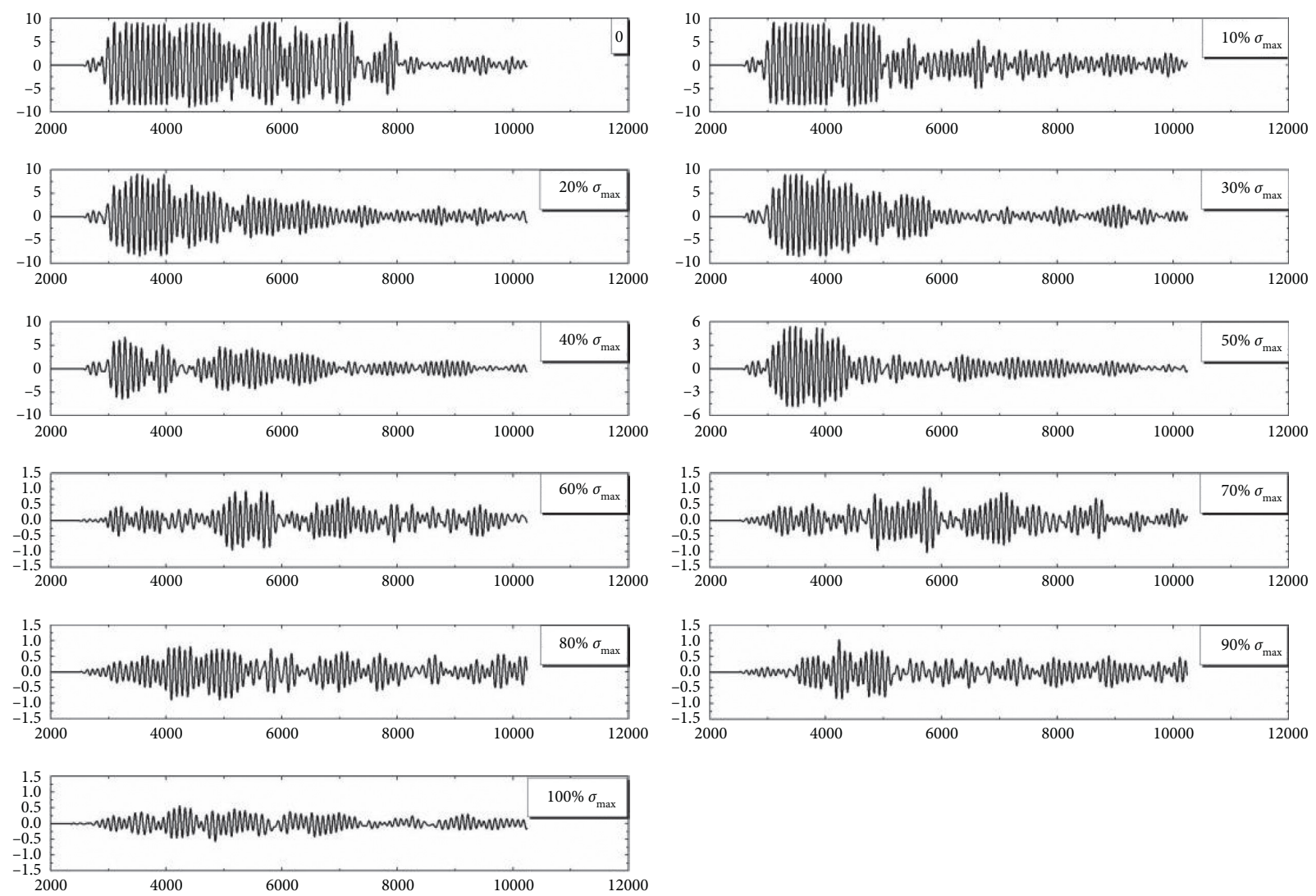

(a)
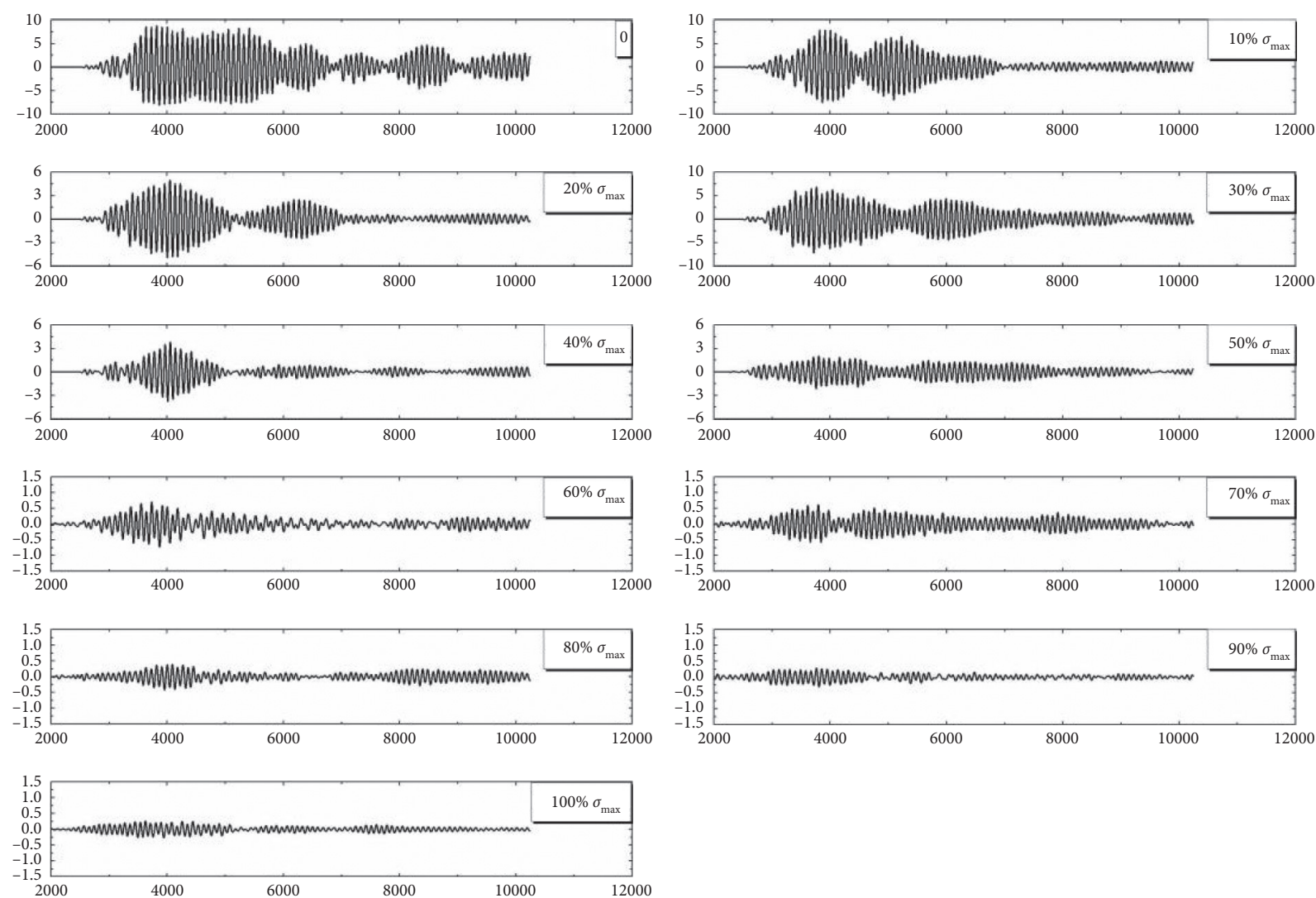

(b)

Figure 3: Continued. 

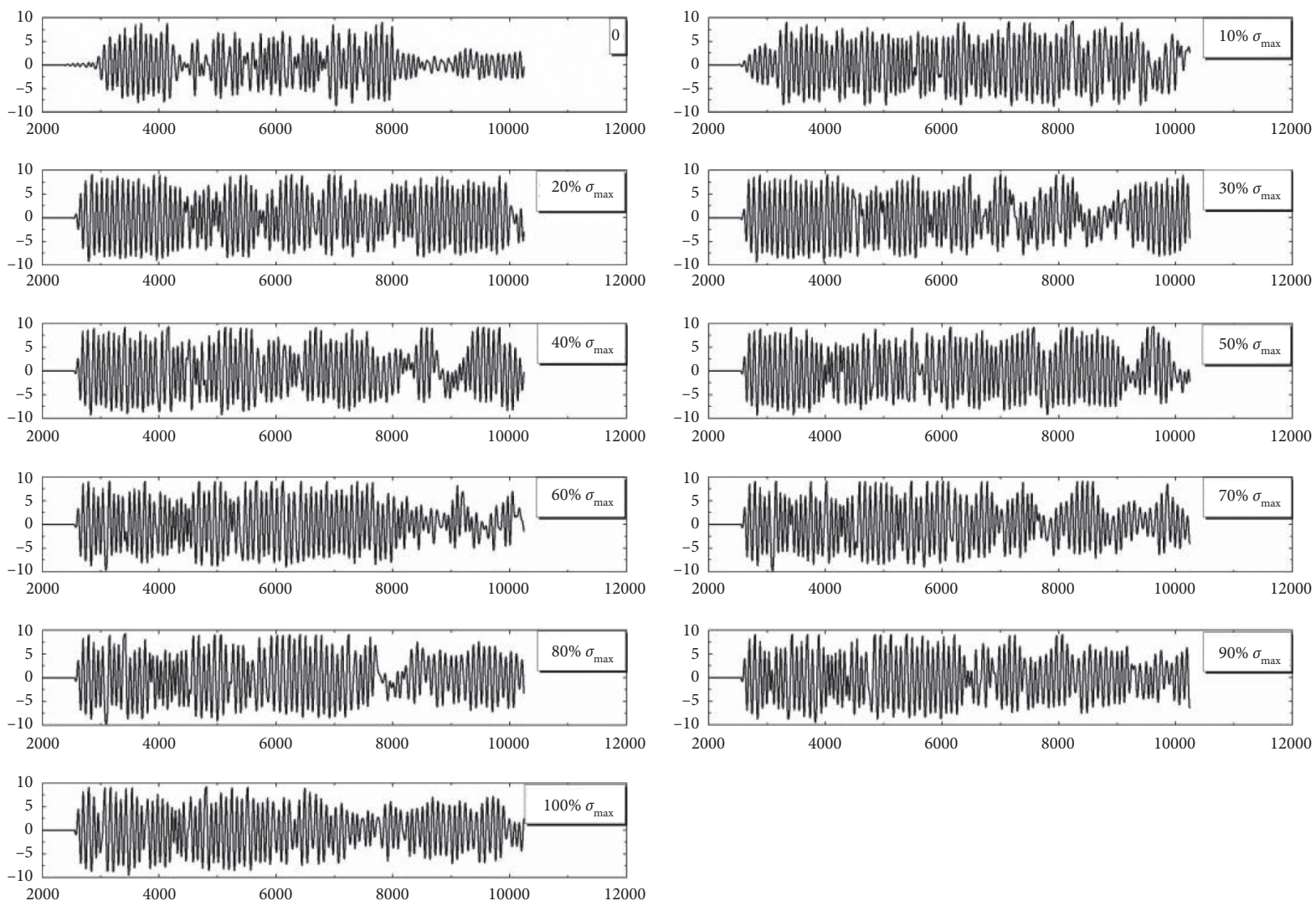

(c)

Figure 3: Receiving waveform at different stress stages. (a) $X$ direction. (b) $Y$ direction. (c) $Z$ direction (the unit of abscissa is $10^{-7}$ s, and the unit of ordinate is V).

can be obtained by calculating the slope of the double logarithmic curve $\ln X_{q}(\varepsilon)-\ln \varepsilon$,

$$
\tau(q)=\lim _{\varepsilon \longrightarrow 0} \frac{\ln X_{q}(\varepsilon)}{\ln \varepsilon} .
$$

Performing the Legendre transform of $\tau(q)-q$, equations (5a) and (5b) can be obtained:

$$
\begin{aligned}
\alpha & =\frac{\mathrm{d}(t(q))}{\mathrm{d} q}=\frac{\mathrm{d}}{\mathrm{d} q}\left(\lim _{\varepsilon \longrightarrow 0} \frac{\ln X_{q}(\varepsilon)}{\ln \varepsilon}\right) . \\
f(\alpha) & =\alpha q-\tau(q) .
\end{aligned}
$$

The curve $\alpha-f(\alpha)$ composed of $\alpha$ and $f(\alpha)$ is the multifractal spectrum of the calculated series, reflecting the unevenly distributed property within frequency spectrum series $\left[x_{i}\right]$.

4.2. Multifractal Spectrum. Based on equation (1), $P_{\max }(\varepsilon)$ corresponds to $\alpha_{\min }$ and $P_{\min }(\varepsilon)$ corresponds to $\alpha_{\max }$. Defined $\Delta \alpha=\alpha_{\max }-\alpha_{\min }$, which reflects the range of probability distribution. The greater the $\Delta \alpha$ is, the wider the multifractal spectrum is and the more irregular the probability distribution is. In this paper, the subsets represented by $\alpha_{\min }$ correspond to a large value, while $\alpha_{\max }$ corresponds to a small value. $\Delta \alpha$ indicates a uniform degree of receiving waveform. The greater the $\Delta \alpha$, the more irregular is the receiving waveform, consequently, the more complex is the receiving waveform.

Defined $\Delta f=f\left(\alpha_{\max }\right)-f\left(\alpha_{\min }\right)$, according to equation (2), equation (6) can be obtained:

$$
f(\alpha)=-\frac{\ln N_{\alpha}(\varepsilon)}{\ln \varepsilon} .
$$

Hence, $\Delta f$ can be expressed as shown in the following relationship:

$$
\begin{aligned}
\Delta f & =-\frac{\ln N_{\alpha_{\max }}-\ln N_{\alpha_{\min }}}{\ln \varepsilon} \\
& =-\frac{\ln N_{P_{\min }}-\ln N_{P_{\max }}}{\ln \varepsilon}=-\frac{\ln \left(N_{P_{\min }} / N_{P_{\max }}\right)}{\ln \varepsilon} .
\end{aligned}
$$

Based on equation (7), it can be found that $\Delta f$ can reflect the proportion of the number of the minimum probability and the maximum probability. In this paper, $\Delta f$ can represent the numerical relationship of vibration amplitude of receiving waveform. $\Delta f>0$ indicates that the small value is dominant, and $\Delta f>0$ indicates that large value is main.

Based on the multifractal theory, the multifractal spectrum $(f(\alpha)-\alpha)$ of receiving waveform in three orthogonal directions were calculated, as shown in Figure 4, and the calculation results showed that receiving waveform had good multifractal characteristics. 

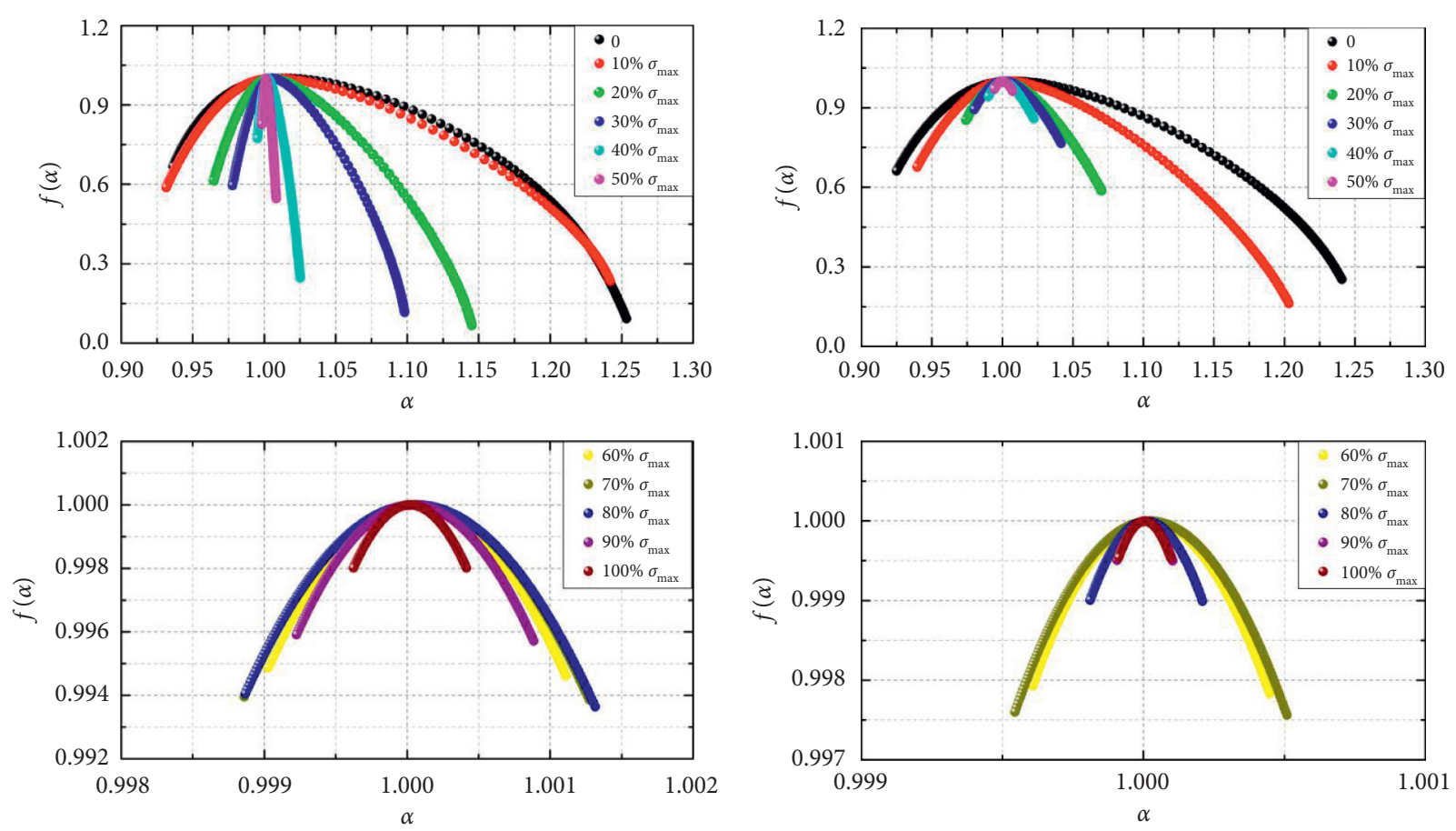

(a)

(b)
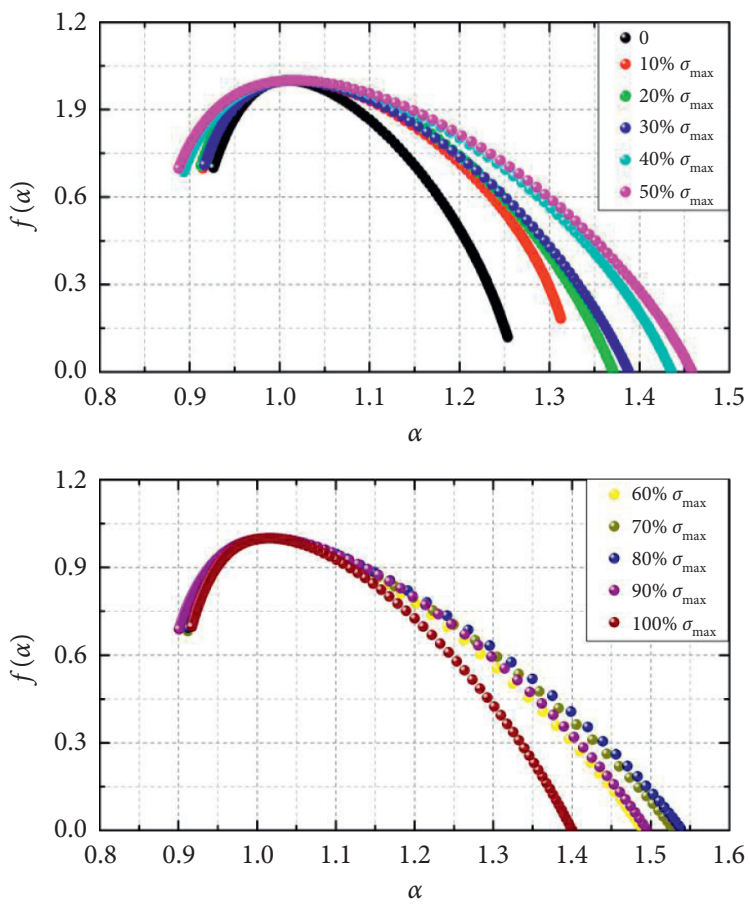

(c)

Figure 4: Multifractal spectrum in three orthogonal directions at the different stress stage. (a) $X$ direction. (b) $Y$ direction. (c) $Z$ direction.

From the perspective of qualitative analysis, the evolution process of the multifractal spectrum of receiving waveform in the $X$ direction and the $Y$ direction was similar during the uniaxial loading process. As the stress increased, the multifractal spectrum presented a trend of getting smaller. However, the evolution process of multifractal spectrum of receiving waveform in the $Z$ direction was notably different. As the stress increased, the multifractal spectrum enlarged slightly before the stress reached $80 \% \sigma_{\max }$. Then, the multifractal spectrum became small until the sample buckling failure.

From the perspective of quantitative analysis, the change trends of multifractal parameters $\Delta \alpha$ and $\Delta f$ with stress are shown in Figure 5. Overall, the multifractal parameters $\Delta \alpha$ in the $X$ direction and the $Y$ direction presented a steady trend 


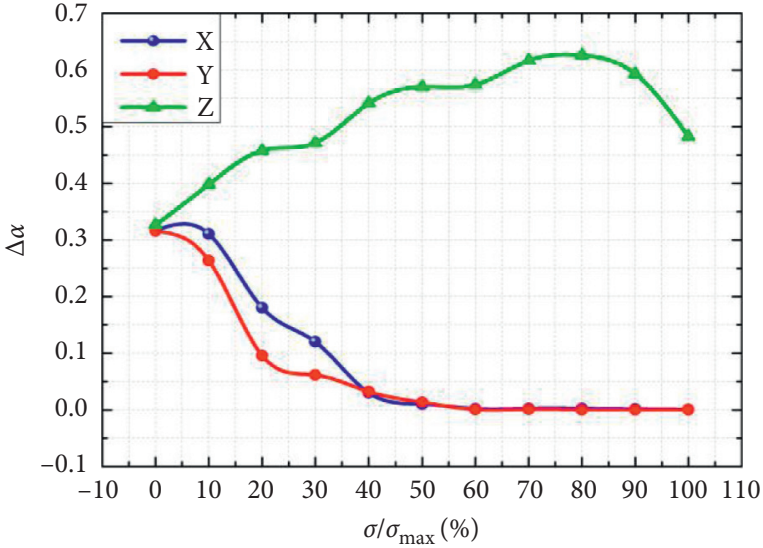

(a)

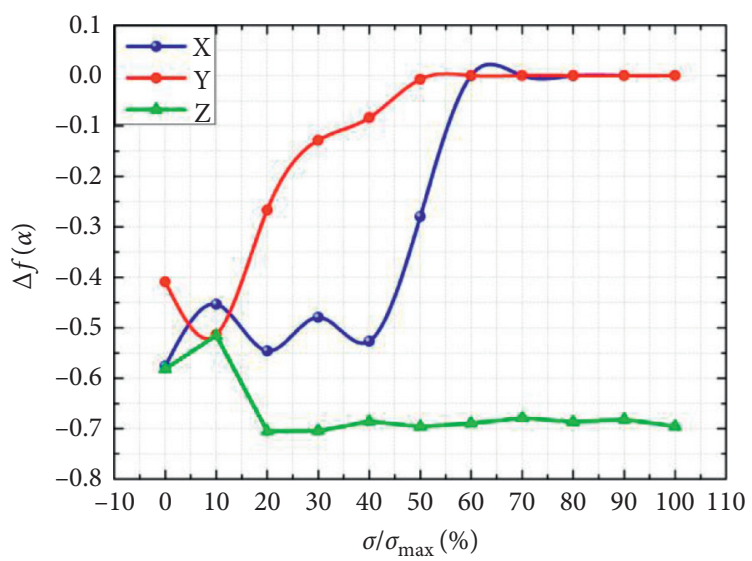

(b)

FIgURE 5: Change of multifractal parameters $\Delta \alpha$ and $\Delta f(\alpha)$ in the different stress stages. (a) Multifractal parameter $\Delta \alpha$. (b) Multifractal parameter $\Delta f(\alpha)$.

after the decline, but the multifractal parameters $\Delta \alpha$ in the $Z$ direction showed a decreasing trend after rising. In contrast, the multifractal parameter $\Delta f$ in the $X$ direction and the $Y$ direction showed a steady trend after rising, but the multifractal parameter $\Delta f$ in the $Z$ direction displayed a steady trend after the decline.

According to Figure 5(a), the multifractal parameters $\Delta \alpha$ in three orthogonal directions were similar before the execution of the loading experiment. At the initial stage of loading $\left(10 \% \sigma_{\max }\right)$, multifractal parameter $\Delta \alpha$ in the $X$ direction and the $Y$ direction was similar and changed less compared with multifractal parameters $\Delta \alpha$ before loading. However, multifractal parameters $\Delta \alpha$ in the $Z$ direction presented an obvious increase trend. When the stress achieved the range of $20 \% \sigma_{\max }-30 \% \sigma_{\max }$, the difference of multifractal parameters $\Delta \alpha$ in three orthogonal directions enlarged. After the stress exceeded $40 \% \sigma_{\max }$, the difference of multifractal parameters $\Delta \alpha$ in the $X$ direction and the $Y$ direction became smaller evidently until reached approximately zero, but multifractal parameters $\Delta \alpha$ in the $Z$ direction increased further. When stress reached $80 \% \sigma_{\max }$, multifractal parameter $\Delta \alpha$ in the $Z$ direction started to decrease until rock sample buckling failure.

Compared with the change trend of multifractal parameter $\Delta \alpha$, the multifractal parameter $\Delta f$ showed the opposite trend. The multifractal parameter $\Delta f$ in the $Z$ direction approximately reached a minimum value when the stress reached $20 \% \sigma_{\max }$, and the multifractal parameter $\Delta f$ in the $X$ direction and the $Y$ direction rise to the maximum value after the stress exceeded $60 \% \sigma_{\max }$. Overall, multifractal parameters $\Delta \alpha$ and $\Delta f$ have good consistency during the entire loading process.

Generally, based on multifractal parameter $\Delta \alpha$, the obtained research results are more accurate and concise. Therefore, for addition support, further analysis is conducted according to the change in multifractal parameter $\Delta \alpha$.

By combining the above analysis results, it is inferred that receiving waveform has clear anisotropic characteristics during the uniaxial loading process reflecting anisotropy of rock properties. The difference of receiving waveform between parallel to the loading direction ( $Z$ direction) and perpendicular to the loading direction ( $X$ direction and $Y$ direction) is large, but the difference of receiving waveform between the $X$ direction and the $Y$ direction is comparatively small. For perpendicular to the loading direction, the difference stage focused on the range $20 \%-40 \%$ of the peak stress.

\section{Evolution Mechanism of Receiving Waveform}

The ultrasonic waveform is transmitted by transmitting terminal, which travels through the rock samples to the receiving terminal. Receiving waveform is formed by superimposition of the multiple waveforms traveling to the receiving terminal following different travel paths. According to the above multifractal theory and calculation results, the more complex is the receiving waveform, the larger is the multifractal parameter $\Delta \alpha$. Generally, combined with the characteristics of ultrasonic waveform, it can be found that the presence of large value waveforms may result in the increase of multifractal parameter $\Delta \alpha$. Corresponding, the obvious increase of small value waveforms may result in the decrease of multifractal parameter $\Delta \alpha$.

The rock samples are processed from limestone with no pronounced cracks; hence, prior to executing the experiment, no obvious anisotropic characteristics are observed. As the aspect ratio of most primary microcracks is small [30], the angle between the microcrack wall and loading direction is large, and the microcracks are easy to close. At the initial stage of loading, the deformation of the rock sample parallel to the loading direction is evident. As the sample becomes denser in the $Z$ direction, the attenuation of the waveform is gradually weakened, and multifractal parameter $\Delta \alpha$ increases gradually. However, the deformation of the rock sample perpendicular to the loading direction is slight, and multifractal parameters $\Delta \alpha$ in the $X$ direction and 
the $Y$ direction slightly change. With increasing stress, microcracks grow gradually along the loading direction [31]. Since the growth direction is almost parallel to the travel direction of the ultrasonic in the $Z$ direction and almost has no impact on the waveform attenuation. The multifractal parameter $\Delta \alpha$ in the $Z$ direction increases further. However, the growth of microcracks affects the amplitude of waveform seriously in the $X$ direction and the $Y$ direction and makes the waveform attenuate obviously. Therefore, multifractal parameter $\Delta \alpha$ in the $X$ direction and the $Y$ direction decreases, and the anisotropic characteristics enlarges reflecting that the difference between rock characteristics parallel to the loading direction and rock characteristics perpendicular to the loading direction become large further. Before the occurrence of the rock samples buckling failure, the rock sample appears macrocrack in large numbers, which causes attenuation of the waveform in the $Z$ direction. This results in multifractal parameter $\Delta \alpha$ in the $Z$ direction start to decrease. At this stage, the rock sample is in a critical state, and anisotropic characteristics weakens.

Xie [17] studied the damage evolution process of the rock samples using the fractal theory during the uniaxial loading process (longitudinal section). The results demonstrated a good linear relationship between the fractal dimension of the damaged area and stress level. In this paper, the multifractal characteristics of receiving waveform are calculated and analyzed. Obviously, receiving waveform is related to damage degree of the rock samples. Noticeably, there is a certain mathematical relationship between multifractal parameter $\Delta \alpha$ of receiving waveform and stress level. According to the data presented in Figure 5, the fitting relationship between multifractal parameter $\Delta \alpha$ and stress can be obtained in three orthogonal directions, as shown in Figure 6. The relationship between multifractal parameter $\Delta \alpha$ of receiving waveform and stress can be described by power function, as expressed in the following equation:

$$
\frac{\sigma}{\sigma_{\max }}=a-b \Delta \alpha^{c}
$$

where fitting parameters $(a, b$, and $c)$ are related to the sample physical and mechanical characteristics, loading model, loading rate, and ultrasonic wave propagation direction.

The analysis from Figures 3-6 revealed that time domain characteristics and multifractal characteristics of receiving waveform in the $X$ direction and the $Y$ direction decrease evidently before stress reaches approximately $60 \% \sigma_{\max }$. With stress continuing to increase, the evolution characteristics of receiving waveform in the $X$ direction and the $Y$ direction change slightly. Although time domain characteristics of receiving waveform in the $Z$ direction almost has no change during the entire loading process, multifractal parameter $\Delta \alpha$ of receiving waveform in the $Z$ direction is very sensitive to stress at the high-stress stage. When stress reaches $80 \% \sigma_{\max }$, evolution characteristics of multifractal parameter $\Delta \alpha$ in the $Z$ direction changes significantly, reflecting transform of damage evolution mechanism.

\section{Intelligence Recognition of Precursory Characteristics of Rock Buckling Failure}

The purpose of studying the multifractal characteristics of receiving waveform in three orthogonal directions is to obtain quantitative relationship between ultrasonic characteristics and damage degree of rock samples, and precursory law of rock samples buckling failure can be recognized further. For precursory characteristics recognition, the intelligence recognition method is generally more efficient and accurate than the traditional human recognition method. Therefore, the backpropagation (BP) neural network is used to recognize precursor characteristics and send early warning signal automatically to reduce misleading and missing alarm phenomenon.

6.1. Basic Theory of the BP Neural Network. The BP neural network is a multilayer feedforward neural network, which is trained using error back propagation algorithm. The basic BP algorithm consists of the forward propagation process and the back propagation process. During the forward propagation process, the output signal is generated by nonlinear transformation of the input signal through the hidden layer. If the output signal does not match the expected results, the error output is backpropagated to the input layer through a hidden layer. During this process, the error is distributed to all units in each layer, and the error signal obtained from each layer is used as the basis for adjusting the weight of each unit. Through adjusting network weight values and bias values, the output results can continuously approach expected results. The topological structure diagram of the BP neural network is shown in Figure 7.

The BP neural network needs to determine weight values and bias values through preliminary training, making it capable of associative memory and prediction capabilities. The training process of the BP neural network includes the following steps:

(1) The parameters need to be determined. Based on characteristics of input signal $X=\left\{x_{1}, x_{2}, \ldots, x_{n}\right\}$ and expected out signal, the number of nodes $n$ in the input layer, the number of nodes $l$ in the hidden layer, and the number of nodes $m$ in the output layer should be determined. The weight values $\left(W_{1}\right.$ and $W_{2}$ ) and bias values ( $B$ and $B^{\prime}$ ) need to be initialized, and incentive function $f(x)$ should be determined.

(2) The output value of the hidden layer is calculated. According to the input signal data, the weight values $W_{1}=\left\{\omega_{11}, \omega_{12}, \ldots, \omega_{n l}\right\}$ and the bias values $B=\left\{b_{1}, b_{2}, \ldots, b_{l}\right\}$. Output value of the hidden layer $H=\left\{h_{1}, h_{2}, \ldots, h_{l}\right\}$ can be calculated as shown in the following equation:

$$
h_{j}=f\left(\sum_{i=1}^{n} \omega_{i j} x_{i}-b_{j}\right) \quad(j=1,2 \cdots, l) .
$$

(3) The output value of the output layer is calculated. According to the output value of the hidden layer $H$, 


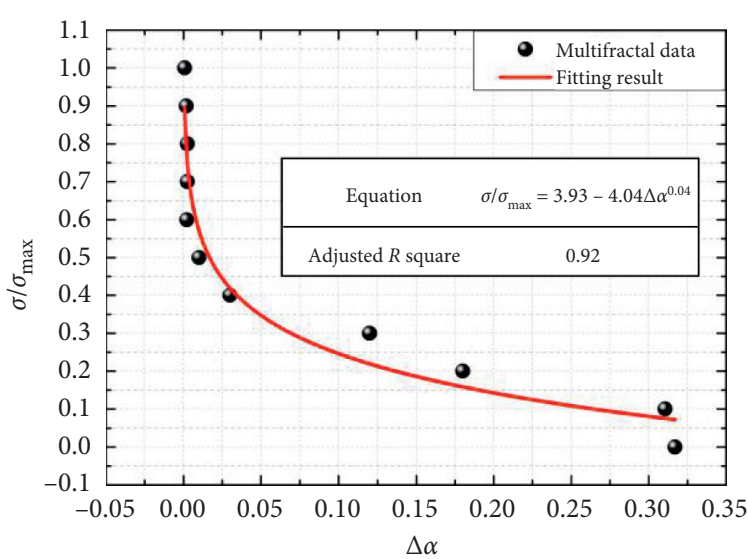

(a)

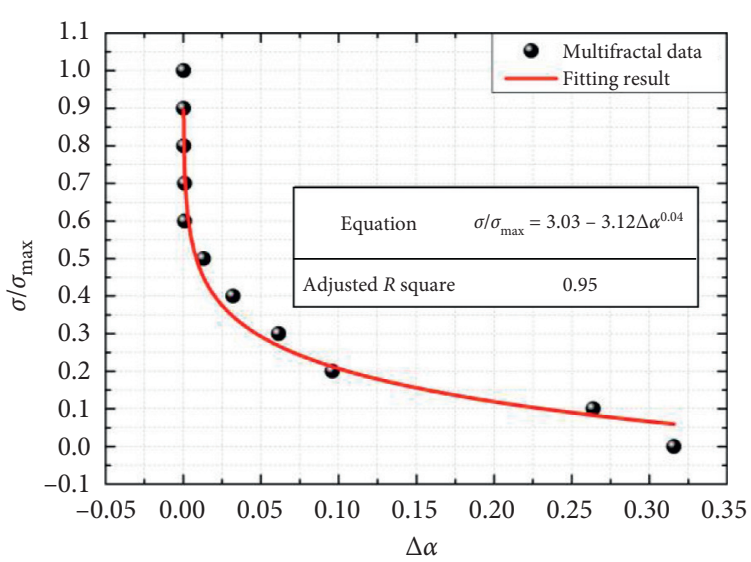

(b)

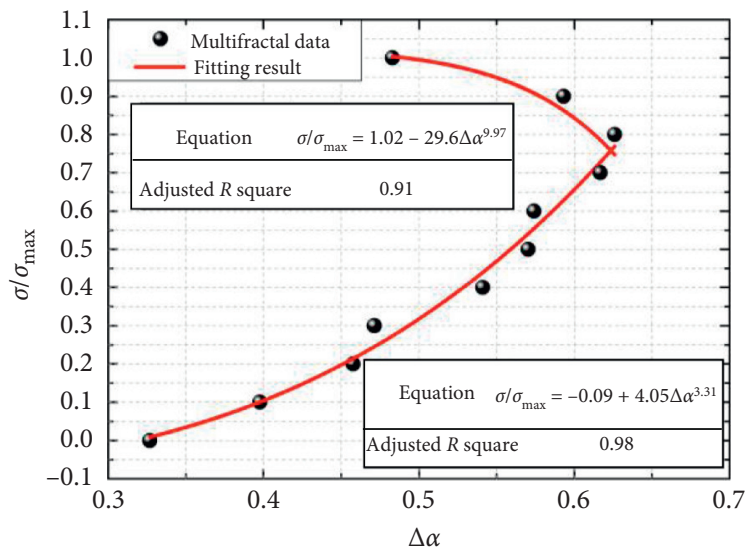

(c)

FIgURE 6: The fitting relationship between multifractal parameter $\Delta \alpha$ and stress. (a) $X$ direction. (b) $Y$ direction. (c) $Z$ direction.

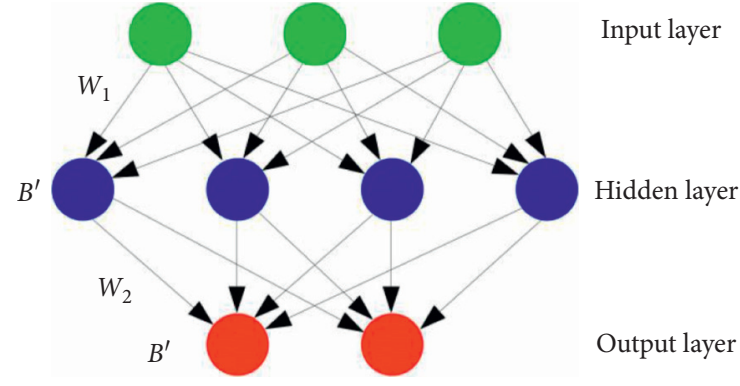

Figure 7: The topological structure diagram of the BP neural network.

the weight values $W_{2}=\left\{\omega_{11}^{\prime}, \omega_{12}^{\prime}, \ldots, \omega_{l m}^{\prime}\right\}$ and bias values $B^{\prime}=\left\{b_{1}^{\prime}, b_{2}^{\prime}, \ldots, b_{m}^{\prime}\right\}$. Output results $C Y=\left\{y_{1}, y_{2}, \ldots y_{m}\right\}$ can be calculated as shown in the following equation:

$$
y_{k}=\sum_{j=1}^{l} \omega_{j k}^{\prime} h_{j}-b_{k}^{\prime}, \quad(k=1,2, \ldots, m) .
$$

(4) Error is calculated. According to the calculation results $C Y$ and real results $R Y=\left\{y_{1}^{\prime}, y_{2}^{\prime}, \ldots y_{m}^{\prime}\right\}$, and the network error $E=\left\{e_{1}, e_{2}, \ldots, e_{m}\right\}$ can be calculated as shown in the following equation:

$$
\left\{\begin{array}{l}
E=\frac{1}{n} \sum_{i=1}^{n} e_{i}, \\
e_{i}=\frac{1}{2} \sum_{k=1}^{m}\left[y_{k}^{\prime}(i)-y_{k}(i)\right]^{2} .
\end{array}\right.
$$

(5) Weight values and bias values are updated. According to error $E$, the weight values and bias values need to be updated, as shown in the following equation:

$$
\left\{\begin{array}{l}
W=W-\alpha \frac{\partial E}{\partial W} \\
B=B-\alpha \frac{\partial E}{\partial B}
\end{array}\right.
$$

where parameter $\alpha$ is learn rate, and the data range is $(0,1)$.

(6) The process of BP algorithm is judged. If the process ends, the output results are generated. If the process does not end, the calculation process is repeated from step (2). 
6.2. Intelligence Recognition of Precursory Characteristics Based on the BPNeural Network. Based on research results in Part 4 and Part 5, a good relationship between multifractal parameters of receiving waveform between stress is observed. It has been proved to be an effective method to describe the damage degree by using ultrasonic technology. In order to recognize precursory characteristics by the BP neural network, the parameters are defined to carry out train. The input data are a four-dimensional matrix, which consists of multifractal parameter $\Delta \alpha$ in three orthogonal directions and the slope of multifractal parameter $\Delta \alpha$ in the $Z$ direction. Taking into account the differences between the samples, the relationship between multifractal parameters waveform between stress is not the same exactly. In order to improve the training effect, the output results are defined as number $0,0.2,0.4,0.6,0.8$, and 1 , representing the ratio between the current stress and the peak stress. All the ultrasonic waveform data of rock samples (from TR-1 to TR12 ) is used to carry out train, including 6271 receiving waveforms in three orthogonal directions. After training, the recognition accuracy of the $\mathrm{BP}$ neural network can reach $96.35 \%$.

In order to verify the predictive effect, the ultrasonic waveform data of VR-1, VR-2, and VR-3 are studied, and precursory characteristics are recognized automatically by the BP neural network. Among them, when the output value first appears 0.8 , the early warning signal is transmitted. Through analysis, the time of early warning of three rock samples is at $1409 \mathrm{~s}, 1588 \mathrm{~s}$, and $1517 \mathrm{~s}$, respectively, which is $175 \mathrm{~s}, 216 \mathrm{~s}$, and $128 \mathrm{~s}$ ahead of the time of buckling failure.

Based on ultrasonic technology, multifractal theory, and BP neural network, a new early warning method of rock buckling failure is proposed, which can provide effective early warning signal before rock buckling failure.

\section{Conclusion}

(1) Advanced ultrasonic measurement device and loading device are used to establish an experiment system for measuring ultrasonic waveform in three orthogonal directions during the uniaxial compression process. The experimental results show that there exists pronounced difference between receiving waveform in the axial direction and radial direction.

(2) Based on the multifractal theory, it is deduced that the receiving waveform in three orthogonal has good multifractal characteristics. The multifractal spectrum of receiving waveform in the radial direction becomes smaller with stress increasing, but the multifractal spectrum in the axial direction present an "increase-decrease" trend.

(3) The analysis of the obtained results of the multifractal parameter $\Delta \alpha$ and $\Delta f$ indicate that the anisotropic characteristics of the rock sample present the trend of "enlarge-reduce" during the entire loading process. Meanwhile, there exists a quantitative relationship between the parameter $\Delta \alpha$ and $\sigma$, which can be expressed by a power function.
(4) Based on the above analysis, a new early warning method is proposed that precursor characteristics of receiving waveform are recognized automatically by BP neural network algorithm. The practical application of the proposed method shows that precursor information can be obtained more than $100 \mathrm{~s}$ in advance before the rock sample buckling failure through the early warning method. This proves the practicability and authenticity of the proposed method.

\section{Data Availability}

The data used to support this study are available from the corresponding author upon request.

\section{Conflicts of Interest}

The authors declare that they have no conflicts of interest.

\section{Acknowledgments}

This work was supported by the Fundamental Research Funds for the Central Universities (FRF-TP-19-025A1) and project funded by China Postdoctoral Science Foundation (2020M670139 and 2018M640533), National Natural Science Foundation of China (51874296), Natural Science Foundation of Jiangsu Province (BK20190080), and State Key Laboratory of Coal Resources and Mine Safety of China (SKLCRSM19X002).

\section{References}

[1] Y. Liu, F. Dai, L. Dong, N. Xu, and P. Feng, "Experimental investigation on the fatigue mechanical properties of intermittently jointed rock models under cyclic uniaxial compression with different loading parameters," Rock Mechanics and Rock Engineering, vol. 51, no. 1, pp. 47-68, 2017.

[2] X. Li, S. Wang, and S. Wang, "Experimental investigation of the influence of confining stress on hard rock fragmentation using a conical pick," Rock Mechanics and Rock Engineering, vol. 51, no. 1, pp. 255-277, 2017.

[3] S. Yang, B. Leshchinsky, K. Cui, F. Zhang, and Y. Gao, "Unified approach toward evaluating bearing capacity of shallow foundations near slope," Journal of Geotechnical and Geoenvironmental Engineering, vol. 145, no. 12, Article ID 04019110, 2019.

[4] S. Yang, B. Leshchinsky, K. Cui, F. Zhang, and Y. Gao, "Influence of failure mechanism on seismic bearing capacity factors for shallow foundations near slopes," Géotechnique, pp. 1-46, 2020.

[5] X. Kong, E. Wang, X. He, E. Zhao, and C. Zhao, "Mechanical characteristics and dynamic damage evolution mechanism of coal samples in compressive loading experiments," Engineering Fracture Mechanics, vol. 210, pp. 160-169, 2019.

[6] E. Wang, X. He, J. Wei, B. Nie, and D. Song, "Electromagnetic emission graded warning model and its applications against coal rock dynamic collapses," International Journal of Rock Mechanics and Mining Sciences, vol. 48, no. 4, pp. 556-564, 2011.

[7] Z. Zhang, E. Wang, N. Li, X. Li, X. Wang, and Z. Li, "Damage evolution analysis of coal samples under cyclic loading based 
on single-link cluster method," Journal of Applied Geophysics, vol. 152, pp. 56-64, 2018.

[8] Y. Zhao and Y. Jiang, "Acoustic emission and thermal infrared precursors associated with bump-prone coal failure," International Journal of Coal Geology, vol. 83, no. 1, pp. 11-20, 2010.

[9] G. Dai, G. Yin, W. Pi, H. Zhu, and C. Xia, CT Real-Time Analysis of Damage Evolution of Coal under Compression, pp. 257-265, CRC Press, Boca Raton, FL, USA, 2004, Xray CT for Geomaterials Ph.D. thesis.

[10] F. Birch, "The velocity of compressional waves in rocks to 10 kilobars: 1," Journal of Geophysical Research, vol. 65, no. 4, pp. 1083-1102, 1960.

[11] A. Nur and G. Simmons, "Stress-induced velocity anisotropy in rock: an experimental study," Journal of Geophysical Research, vol. 74, no. 27, pp. 6667-6674, 1969.

[12] Z. Zhang, E. Wang, D. Chen, X. Li, and N. Li, "The observation of $\mathrm{AE}$ events under uniaxial compression and the quantitative relationship between the anisotropy index and the main failure plane," Journal of Applied Geophysics, vol. 134, pp. 183-190, 2016.

[13] M. Q. Guo and L. Y. Fu, "Stress associated coda attenuation from ultrasonic waveform measurements," Geophysical Research Letters, vol. 34, no. 9, 2007.

[14] A.-M. Wulff, T. Hashida, K. Watanabe, and H. Takahashi, "Attenuation behaviour of tuffaceous sandstone and granite during microfracturing," Geophysical Journal International, vol. 139, no. 2, pp. 395-409, 1999.

[15] J. Cao, Z.-H. He, and D. J. Huang, "Physical modeling and Ultrasonic experiment of pore-crack in reservoirs," Progress in Geophysics, vol. 19, no. 2, pp. 386-391, 2004.

[16] M. N. Toksöz, D. H. Johnston, and A. Timur, "Attenuation of seismic waves in dry and saturated rocks: I. Laboratory measurements," Geophysics, vol. 44, no. 4, pp. 681-690, 1979.

[17] H. Xie, Introduction to Fractal Rock Mechanics, Science Press, Beijing, China, 1996.

[18] L. Shan, S. Hu, Y. Zhang et al., "Relationship between fractal meso-structural and mechanical characteristics of lump coal under uniaxial compression at different temperatures," Fuel Processing Technology, vol. 194, Article ID 106112, 2019.

[19] X. Li, M. Luo, and J. Liu, "Fractal characteristics based on different statistical objects of process-based digital rock models," Journal of Petroleum Science and Engineering, vol. 179, pp. 19-30, 2019.

[20] S. Hu, E. Wang, Z. Li, R. Shen, and J. Liu, "Time-varying multifractal characteristics and formation mechanism of loaded coal electromagnetic radiation," Rock Mechanics and Rock Engineering, vol. 47, no. 5, pp. 1821-1838, 2014.

[21] B. Kong, E. Wang, Z. Li, and W. Lu, "Study on the feature of electromagnetic radiation under coal oxidation and temperature rise based on multifractal theory," Fractals, vol. 27, no. 3, Article ID 1950038, 2019.

[22] Z. Zhang, E. Wang, and N. Li, "Fractal characteristics of acoustic emission events based on single-link cluster method during uniaxial loading of rock," Chaos, Solitons \& Fractals, vol. 104, pp. 298-306, 2017.

[23] J. Pei, W. Fei, and J. Liu, "Spatial evolution and fractal characteristics of natural fractures in marbles under uniaxial compression loading based on the source location technology of acoustic emission," Environmental Earth Sciences, vol. 75, no. 9, p. 828, 2016.

[24] H. P. Xie, J. F. Liu, Y. Ju, J. Li, and L. Z. Xie, "Fractal property of spatial distribution of acoustic emissions during the failure process of bedded rock salt," International Journal of Rock
Mechanics and Mining Sciences, vol. 48, no. 8, pp. 1344-1351, 2011.

[25] Z. Zhang, E. Wang, Y. Zhang et al., "Analysis on the timefrequency characteristics of ultrasonic waveform of coal under uniaxial loading," Fractals, vol. 27, no. 6, Article ID 1950100, 2019.

[26] X. Sun, Z. Fu, and Z. Wu, "Multifractal analysis and scaling range of ZnO AFM images," Physica A: Statistical Mechanics and its Applications, vol. 311, no. 3-4, pp. 327-338, 2002.

[27] G. Xiong, W. Yu, and S. Zhang, "Singularity power spectrum distribution," Physica A: Statistical Mechanics and Its Applications, vol. 431, pp. 63-73, 2015.

[28] Z. Zhang, E. Wang, E. Zhao, and S. Yang, "Nonlinear characteristics of acoustic emission during the heating process of coal and rock," Fractals, vol. 26, no. 4, Article ID 1850046, 2018.

[29] D. A. Aouit and A. Ouahabi, "Nonlinear fracture signal analysis using multifractal approach combined with wavelets," Fractals, vol. 19, no. 2, pp. 175-183, 2011.

[30] A. Ougier-Simonin, J. Fortin, Y. Guéguen, A. Schubnel, and F. Bouyer, "Cracks in glass under triaxial conditions," International Journal of Engineering Science, vol. 49, no. 1, pp. 105-121, 2011.

[31] J. Xu, H. Li, X. Xian, and G. Yin, "Experimental study on the entire process of microscopic fracture development of sandstone under uniaxial stress," Mechanics in Engineering, vol. 8, no. 4, pp. 18-22, 1986. 